\title{
Effects of prosthetic foot forefoot flexibility on gait of unilateral transtibial prosthesis users
}

Elizabeth Klodd, MS; ${ }^{1}$ Andrew Hansen, PhD; ${ }^{2-3 *}$ Stefania Fatone, PhD, BPO(Hons); ${ }^{3}$ Mark Edwards, MHPE, CP ${ }^{3-4}$ ${ }^{1}$ Liberating Technologies Inc, Holliston, MA; ${ }^{2}$ Minneapolis Department of Veterans Affairs Health Care System, Minneapolis, MN; ${ }^{3}$ Prosthetics-Orthotics Center, Department of Physical Medicine \& Rehabilitation, Feinberg School of Medicine, Northwestern University, Chicago, IL; ${ }^{4}$ Otto Bock HealthCare, Minneapolis, MN

\begin{abstract}
Five solid-ankle experimental prosthetic feet were used in this double-blind randomized crossover study to determine the effects of forefoot flexibility on gait of 14 unilateral transtibial prosthesis users. Flexibility in experimental feet was altered by changing the number of flexural hinges in their forefoot sections. When experimental prosthetic foot conditions were compared, measured prosthetic ankle dorsiflexion range of motion increased as much as $3.3^{\circ}$ with increasing flexibility $(p<0.001)$ and the foot's anterior moment arm (measured as the effective foot length ratio) increased as much as $23 \%$ of the foot length with decreasing flexibility $(p<0.001)$. Subjects also showed increases in the difference between sound and prosthetic ankle moments as high as $0.53 \mathrm{Nm} / \mathrm{kg}$ in late stance phase of walking as flexibility decreased $(p<0.001)$. The difference between first peaks of the vertical ground reaction forces on the sound and prosthetic sides increased as much as $9 \%$ of body weight when subjects used the foot with the greatest flexibility $(p=0.001)$. The results of this study suggest solid-ankle prosthetic foot designs with overly flexible forefoot sections can cause a "drop-off" effect in late stance phase and during the transition of loading between prosthetic and contralateral limbs.
\end{abstract}

Key words: ambulation, amputee, artificial limb, foot, gait, leg, lower limb, prosthesis, rehabilitation, walking.

\section{INTRODUCTION}

Although a large number of prosthetic foot types are commercially available, clinically useful objective evidence to guide their prescription is scarce [1-3]. At the same time, the field of prosthetics is changing rapidly. Many new devices are introduced each year and many leave the market, making it is difficult for clinicians to choose the best possible components for their patients with amputations. This problem is certainly true of prosthetic ankle-foot systems. The rapid change in the available foot types supports the need for an improved core understanding of prosthetic foot properties and their effects on user function.

Earlier studies have attempted to determine differences between groups of commercially available prosthetic feet to aid in understanding and prescribing these systems [4]. However, the feet in these groups normally have many mechanical differences, which makes linking a functional difference to a specific prosthetic foot feature difficult. Also, many of the feet that have been tested in the past are no longer commercially available or have

Abbreviations: $\mathrm{AM}=$ ankle moment, $\mathrm{AMD}=$ ankle moment difference, ANOVA = analysis of variance, EFLR = effective foot-length ratio, GRF = ground reaction force, $\mathrm{ROM}=$ range of motion, SL = step length, SLD = step-length difference, VA = Department of Veterans Affairs, VGRF = vertical ground reaction force.

* Address all correspondence to Andrew Hansen, PhD; Minneapolis VA Health Care System, One Veterans Drive (151), Minneapolis, MN 55417; 612-467-2910.

Email: Andrew.Hansen2@va.gov

DOI:10.1682/JRRD.2009.10.0166 
been altered by their manufacturers through periodic design changes. A more controlled approach is needed to broaden our core understanding of prosthetic foot features and their effects on function of the prosthesis user.

Prosthetic feet have many features, including energy storage and return, hysteresis, effective foot length (anterior moment arm), and general stiffness properties. Many of these properties are not independent-e.g., stiffness and hysteresis relate to the amount of energy storage and return possible with the foot. This interdependence of mechanical properties causes difficulty in understanding which properties lead to specific gait deviations when they occur. Controlled studies that attempt to change only one feature at a time are needed to help build a core knowledge of these features and their effects on amputee gait.

One area in which a more controlled approach to research is needed is in the study of prosthetic ankle-foot flexibility and its effects on gait. Previous studies have reported decreased sound-limb loading in unilateral transtibial prosthesis users when they used the Flex-Foot, a Jshaped prosthetic foot with flexibility along its length from the socket to the end of the foot (Össur; Reykjavik, Iceland). These studies also measured an increased "ankle" range of motion (ROM) on the prosthetic side when subjects used the Flex-Foot [5-7]. One may presume causality between these findings and conclude that prosthetic ankle-foot systems offering more flexibility will reduce sound-limb loading. However, this idea may be problematic if taken to extremes. For example, prosthetic feet that have extreme levels of forefoot flexibility may not allow adequate forward progression of the center of pressure of the ground reaction force (GRF) during late stance on the prosthetic side, thus decreasing the prosthetic foot's effective foot length. A person using a prosthetic foot with a short effective foot length may experience a reduced ankle moment on the prosthetic side and a "drop-off" effect during load transfer from the prosthesis to the sound limb. Possible consequences of the drop-off effect include shorter step lengths on the sound side, increased vertical GRFs (VGRFs) on the sound limb during initial stance phase, and decreased VGRFs on the prosthetic limb during the end of stance phase. Hansen et al. altered the effective keel length of an experimental prosthetic foot and observed a drop-off effect when subjects used prosthetic feet with smaller effective foot lengths, including increased loading on the sound side [8]. Prosthetic feet that are overly flexible likely will not allow forward progression of the GRF under the foot, yielding similar results as found when the keel structure is shortened (Figure 1).

The purpose of this study was to investigate the effects of forefoot flexibility on gait of unilateral transtibial prosthesis users using an experimental solid-ankle foot in which the forefoot flexibility feature could be easily altered without changing other properties of the feet. We hypothesized that-

1. Measured "ankle" flexion on the prosthetic side would increase with increasing forefoot flexibility.

2. Effective foot-length ratios (EFLRs - defined by Hansen et al. as the distance from the heel of the foot to the center of pressure location in foot coordinates at opposite heel contact, divided by the overall length of the foot [9])

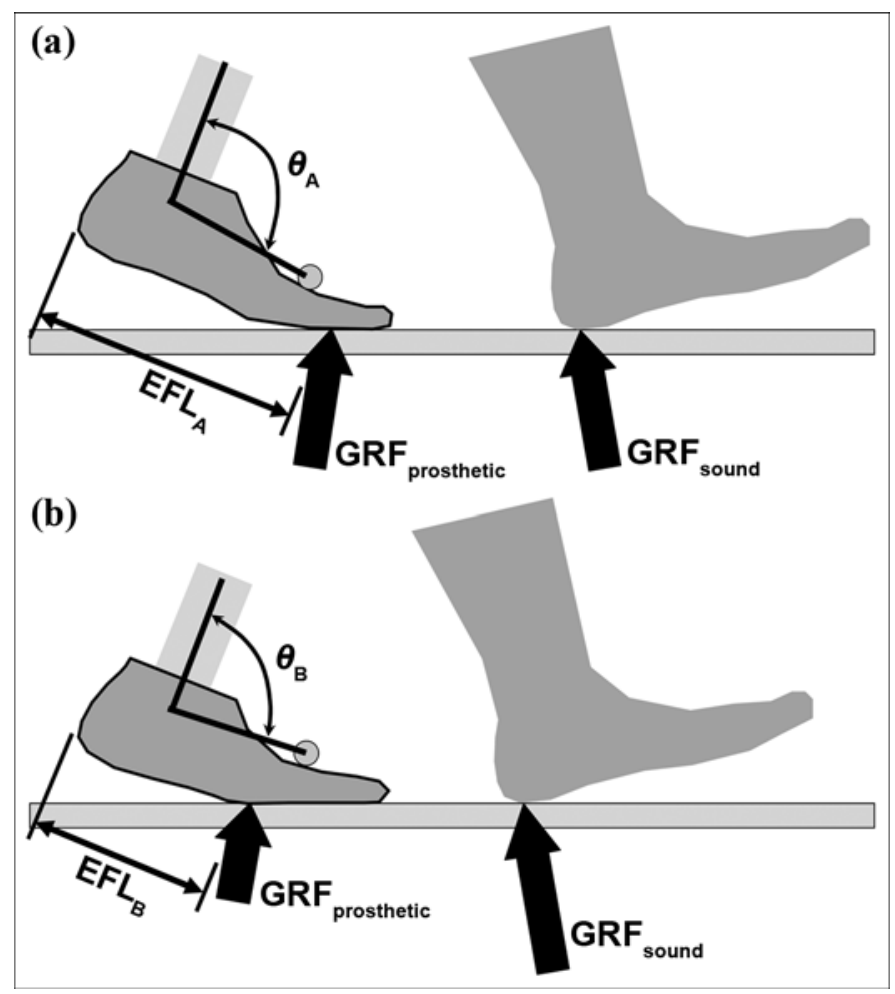

Figure 1.

Hypothesized differences between prosthetic feet with different levels of flexibility at time of opposite initial contact: (a) prosthetic foot that allows long forward progression of ground reaction force (GRF) and (b) highly flexible prosthetic foot with limited forward progression of GRF. Effective foot length (EFL) of foot A is larger than that of foot B. "Ankle" flexion $(\theta)$ range of motion for foot $\mathrm{B}$ is larger than for foot A. "Drop-off” in GRF is expected on overly flexible feet (such as foot B), leading to increased initial loading on sound limb and potentially reduced sound limb step length. 
on the prosthetic side would decrease with increasing forefoot flexibility.

3. Late-stance ankle moments on the prosthetic side would decrease with increasing forefoot flexibility.

4. Prosthetic feet with excessive forefoot flexibility would lead to increased sound-limb loading, due to a "drop-off" effect.

The results of this study should add to the core knowledge regarding prosthetic foot features and their effects on gait, making them directly relevant to prosthesis design and prescription.

\section{METHODS}

\section{Data Collection}

Subjects with unilateral transtibial amputation were recruited to participate in this double-blind randomized crossover trial. Subjects who had participated in prior experiments in our laboratory and who had indicated willingness to be contacted for future research were contacted. Additionally, approved fliers were posted in local hospitals and were distributed to a number of local clinicians for distribution to their clients who might be interested in the research project. All subjects who were interested in the study completed an informed consent process and signed the approved consent form. Recruitment criteria included having a minimum of 1 year of experience walking on a definitive prosthesis, having the ability to walk without the use of assistive devices such as canes or walkers, and being between 18 and 80 years old.

After consent was obtained during the first visit, the height, weight, and foot length of each subject were mea- sured and recorded. Next, five versions of the Shape\&Roll prosthetic foot were fabricated according to the procedures described by Sam et al. [10]. Each version was cut and sanded to fit inside a commercially available foot shell that corresponded to the user's intact foot size. All five prosthetic feet were identical except for their forefoot flexibilities (labeled F1 to F5, with F1 being most flexible and F5 being least flexible). A band saw was used to make a different number of cuts in the forefoot of each of the five feet corresponding to different flexibilities. The number and placement of the cuts were determined by a MATLAB (Mathworks Inc; Natick, Massachusetts) program that estimated different amounts of forefoot bending in an attempt to achieve different overall amounts of forefoot flexion, within the range of flexibilities found in commercially available prosthetic feet [11]. In particular, the F3 prosthetic foot was designed to conform to a roll-over shape matching the nondisabled ankle-foot system in walking [12]. Post hoc testing of size $24 \mathrm{~cm}$ experimental feet in a materials testing machine (MTS; Eden Prairie, Minnesota) at a loading angle of $20^{\circ}$ on the forefoot yielded $28 \mathrm{~mm}$ deflection at 1,000 $\mathrm{N}$ for the $\mathrm{F} 1$ foot and $18 \mathrm{~mm}$ deflection at $1,000 \mathrm{~N}$ for the $\mathrm{F} 5$ foot. After the five feet had been fabricated, they were ready to be aligned for each subject during the second visit (Figure 2).

A qualified prosthetist performed static and dynamic alignments of the prostheses for each subject's usual socket. The same prosthetist aligned all the feet for each subject. The subject's usual prosthesis was disconnected at the socket/pylon pyramid attachment in a manner that preserved the alignment of the usual prosthesis. The prosthetist preserved the alignment of each prosthetic foot by backing off two adjacent nonprotruding attachment screws
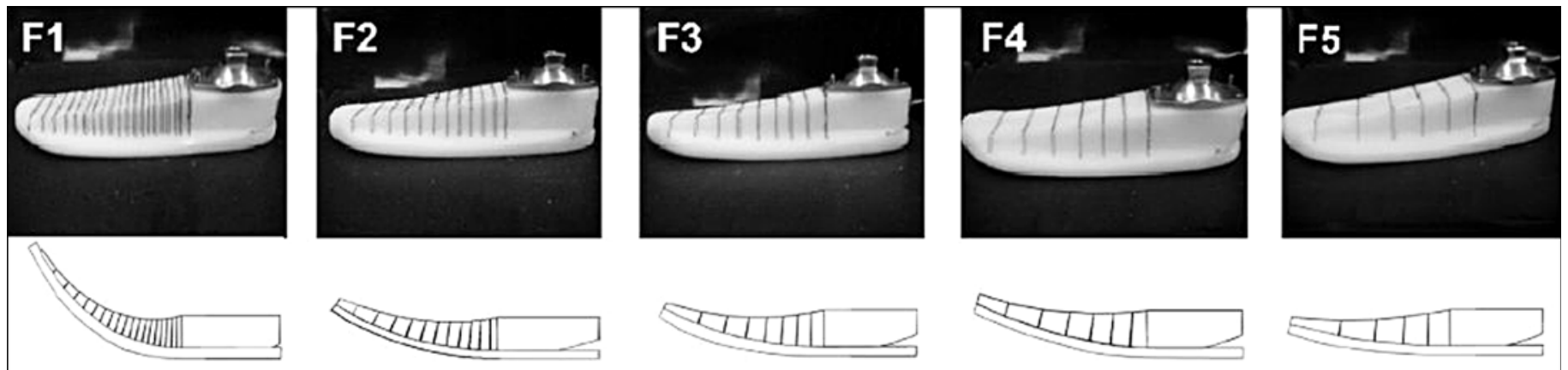

Figure 2.

Five Shape\&Roll prosthetic feet, shown left to right in order of decreasing flexibility (i.e., F1 is most flexible and F5 is least flexible). Flexibility was achieved with use of flexural hinges in forefoot region of feet. Increasing number of hinges in series created more flexible prosthetic feet. 
at the socket pyramid attachment. The order in which the five experimental prosthetic feet (F1, F2, F3, F4, and F5) were aligned was assigned randomly with the use of dice. Subjects wore their own shoes with each prosthetic foot condition, and the same shoes were also used during gait analyses.

The hardware used to connect the prosthetic feet to the sockets included an Otto Bock 2R57 (Otto Bock HealthCare; Minneapolis, Minnesota) pylon with a 4R88 sliding adapter at the socket attachment point and a pyramid adapter at the distal end. The same foot shell was used for each of the five experimental feet. Also, a sock was used between the prosthetic foot core and the foot shell for minimizing movement between the foot shell and the core, and blinding the prosthetist and subject to the different foot designs. Once alignment was completed, subjects walked a total of five laps around a series of hallways in the laboratory (a total distance of approximately $435 \mathrm{~m}$ ) to familiarize themselves with each foot. All walking was done on a level tile surface. If the subject or prosthetist felt additional changes in alignment were needed after the five laps, the prosthetist made adjustments accordingly.

After the alignment process, each subject returned to the laboratory for a quantitative gait analysis. During this third visit, subjects walked with each of the five experimental feet with their usual prosthetic socket. For all subjects, the same study personnel placed the reflective markers according to a modified Helen Hayes marker set [13]. This marker set was used for the torso and sound limb. Because a prosthesis was used, the prosthetic side marker placement (Figure 3) was modified.

First, a marker was placed laterally on the socket at a position that estimated the knee center of rotation. A sec-
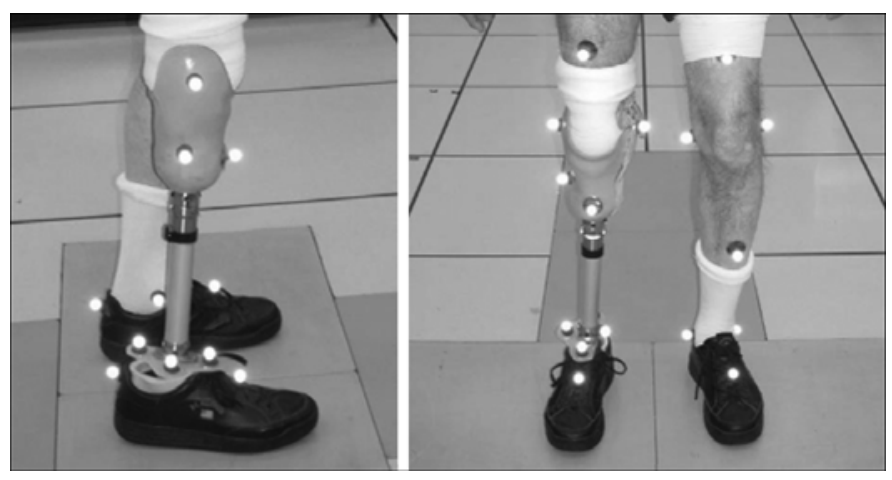

Figure 3.

Pictures showing marker placement on prosthesis and intact limb of representative subject. ond marker was placed on the distal-lateral aspect of the socket. A third marker was placed on the distal-anterior aspect of the socket. On all prosthetic feet, one marker was placed posteriorly on the heel and another "toe marker" on the dorsal aspect of the forefoot, immediately proximal to the usual location of the metatarsophalangeal joint. Heel and toe markers were placed on the shoe, not the foot itself. Three markers were placed on an ankle plate that was secured between the foot and pylon, projected laterally from the prosthesis, and was parallel to the attachment surface of the foot. Markers were placed on the lateral, anterior, and posterior sides of the plate. The lateral marker on the plate served as the "ankle" marker for the prosthesis, because this position was easily standardized between the different foot conditions. For static trials, additional medial markers were placed on the socket at a position that estimated the medial knee center of rotation and at a location medial to the lateral ankle marker on the prosthetic side (Figure 3).

Once all the markers were placed on the subject and the prosthesis, a static trial was conducted with medial knee and ankle markers attached that estimated joint centers for the sound side and for the knee joint on the prosthetic side. After the static trial, the medial markers were removed and the subject walked at a normal self-selected speed along a 10 m walkway. Eight Eagle Digital Real-Time motion analysis cameras (Motion Analysis Corporation; Santa Rosa, California) recorded movements of the reflective markers at $120 \mathrm{~Hz}$, and six force plates (Advanced Mechanical Technology, Inc; Watertown, Massachusetts) embedded in the floor measured GRFs at $960 \mathrm{~Hz}$ as the subject walked. Force plate data were later resampled in software at $120 \mathrm{~Hz}$ to synchronize the data with marker trajectory data. Trials were collected until subjects produced four "clean" force plate hits on each side. A clean force plate hit was one in which the foot landed on a force plate without crossing the plate's boundaries and without the other foot touching that particular force plate during the trial.

After this process was completed with the first experimental prosthetic foot, the prosthesis was doffed and the same protocol repeated for each of the four remaining experimental feet. The order in which the feet were used was determined by random selection (with dice) and was potentially different than the order used in the alignment process. Static data collection and walking trials were repeated for each of the foot conditions. 


\section{Data Processing and Analysis}

Three-dimensional motion data were processed in EVa RealTime Version 5.0 (Motion Analysis Corporation). Small gaps of missing data points were joined with cubic spline interpolation techniques. Curves were smoothed with a second-order bidirectional filter with an effective cutoff frequency of $6 \mathrm{~Hz}$ [14]. These data were then processed in Orthotrak Version 6.5.2 (Motion Analysis Corporation). This software calculated gait events and joint moments, powers, and angles. MATLAB and Microsoft Excel (Microsoft Corporation; Redmond, Washington) were then used to plot data.

The following variables, representing a difference between the prosthetic and sound sides, were analyzed in this study:

- Step length difference:

$$
\mathrm{SLD}=\mathrm{SL}_{\text {prosthetic side }} \angle \mathrm{SL}_{\text {sound side }} \text {, }
$$

where SLD = step length difference and SL = step length.

- First peak of the VGRF difference:

$\operatorname{VGRF}($ force $/$ weight $)=\max \left(\frac{1 \text { stpeakVGRF }_{\text {sound side }}}{\text { weight }}\right)<\max \left(\frac{1 \text { stpeakVGRF }_{\text {rrostheic side }}}{\text { weight }}\right)$,

where 1 st peak = first peak.

- Peak ankle moment difference:

$$
\operatorname{AMD}(\mathrm{Nm} / \mathrm{kg})=\max \left(\mathrm{AM}_{\text {sound side }}\right) \angle \max \left(\mathrm{AM}_{\text {prosthetic side }}\right)
$$

where AMD = ankle moment difference and AM = ankle moment.

We analyzed the differences of these variables as opposed to the actual values to compare symmetry. The step length differences were determined as the average of prosthetic side step length for each trial minus the average of sound side step lengths for the trial. The overall difference for step length for each subject was then determined as the average of these differences across trials. We found force and moment differences by first calculating average forces and moments on the sound side from all trials and subtracting from the average forces and moments on the prosthetic side for all trials. The different techniques for forces and moments compared with step length stemmed from all trials not having clean force plate hits from both sides of the body.

Walking speed, the EFLR, and prosthetic ankle ROM were also analyzed. We determined the EFLR from the rollover shape [9], which was calculated by transforming the center of pressure from the laboratory-based coordinate system into a coordinate system formed by the three markers on the ankle plate. "Ankle" motion on the prosthetic side was calculated as the angle between one vector extending from the ankle center to the knee center and another vector from the ankle center to the forefoot marker (Figure 1). We chose this method instead of a method using a heel marker to reduce the effects of measurement error due to the heel of the foot slipping within the foot shell. This method produces angles that are shifted from those that would be reported clinically. For example, a "neutral" ankle angle as reported clinically would be greater than $90^{\circ}$ as measured in this study (Figure 1). However, we chose this method for consistency between the experimental feet and to instead explore if the range of ankle motion was changed as forefoot flexibility increased to compare results with previous literature [5-7].

Data were analyzed with six one-way repeated measures analysis of variance (ANOVA) with $n=14$ and a significance level of $p=0.05$ (one repeated measures ANOVA for each of the six variables). For factors that were significantly different at $\alpha<0.05$, pairwise comparisons (paired $t$-tests) were made with the use of Bonferroni adjustments for multiple comparisons. The software automatically adjusted to $\alpha=0.05$ after the Bonferroni adjustments. The assumptions of repeated measures ANOVA are that the data are normally distributed and that they have sphericity (a term relating to equal variances in different dimensions). Data were checked for normality with the Shapiro-Wilk Test of Normality. Since we had a total of 5 foot conditions and 6 variables, 30 data sets were tested for normality. We used Mauchly's Test of Sphericity to examine the assumption of sphericity. If the data were normally distributed but the assumption of sphericity was violated, the Greenhouse-Geisser correction factor was used. All statistical tests were performed with SPSS 16.0 (SPSS, Inc; Chicago, Illinois). Our approach to statistics is consistent with that described by Field [15].

\section{RESULTS}

A total of 14 unilateral transtibial prosthesis users completed the study. The average age of the subjects was $53 \pm 11$ years (mean \pm standard deviation). The average height was $170 \pm 10 \mathrm{~cm}$ and the average mass was $86 \pm$ $11 \mathrm{~kg}$. Additional subject data are shown in Table 1. 
JRRD, Volume 47, Number 9, 2010

All subjects regularly ambulated without the use of assistive devices and had at least 1 year of experience walking on a prosthetic limb. Thirty data sets are reported in this section for the five feet and the six variables of interest. All 30 data sets were found to be normally distrib- uted, supporting the use of parametric statistical analysis. Results of statistical testing are outlined in Table 2.

Walking speed was not significantly affected by flexibility ( $p=0.153$ ). A main effect for step length differences across the five feet was found ( $p=0.029$ );

Table 1.

Subject data.

\begin{tabular}{|c|c|c|c|c|c|c|c|}
\hline Subject & Sex & Age & $\begin{array}{l}\text { Height } \\
\text { (cm) }\end{array}$ & $\begin{array}{l}\text { Weight } \\
\text { (kg) }\end{array}$ & Usual Foot & Suspension & $\begin{array}{l}\text { Reason for } \\
\text { Amputation }\end{array}$ \\
\hline 1 & $\mathrm{M}$ & 65 & 185.0 & 88.8 & $\begin{array}{l}\text { Otto Bock Springlite } \\
\text { (Otto Bock Healthcare; Minneapolis, MN) }\end{array}$ & Supracondylar & Trauma \\
\hline 2 & $\mathrm{M}$ & 50 & 183.0 & 110.0 & $\begin{array}{l}\text { Össur Vari-Flex } \\
\text { (Össur; Reykjavik, Iceland) }\end{array}$ & Pin & Trauma \\
\hline 3 & F & 45 & 162.5 & 63.8 & Össur Ceterus & Pin & Trauma \\
\hline 4 & $\mathrm{~F}$ & 59 & 162.0 & 96.0 & $\begin{array}{l}\text { Seattle Lightfoot } \\
\text { (Seattle Systems; Poulsbo, WA) }\end{array}$ & Pin & Trauma \\
\hline 5 & $\mathrm{~F}$ & 49 & 158.0 & 83.5 & Seattle Lightfoot & Sleeve & Lupus \\
\hline 6 & F & 50 & 158.0 & 87.0 & $\begin{array}{l}\text { Össur Elation } \\
\text { (Össur; Reykjavik, Iceland) }\end{array}$ & Pin & Trauma \\
\hline 7 & M & 57 & 177.0 & 99.0 & $\begin{array}{l}\text { Freedom Renegade } \\
\text { (Freedom Innovations; Irvine, CA) }\end{array}$ & Pin & Trauma \\
\hline 8 & F & 48 & 166.0 & 78.0 & $\begin{array}{l}\text { College Park TruStep } \\
\text { (College Park Industries; Fraser, MI) }\end{array}$ & Sleeve & Trauma \\
\hline 9 & $\mathrm{~F}$ & 63 & 157.0 & 83.5 & $\begin{array}{l}\text { Endolite Multiflex } \\
\text { (Endolite; Centerville, OH) }\end{array}$ & Sleeve & Spina Bifida \\
\hline 10 & $\mathrm{M}$ & 68 & 170.0 & 76.5 & Seattle Lightfoot & Pin & Infection \\
\hline 11 & M & 50 & 177.0 & 89.0 & Flex-Foot Flex-Walk & Sleeve & PVD \\
\hline 12 & $\mathrm{M}$ & 51 & 176.0 & 79.0 & Seattle Lightfoot & Sleeve & Trauma \\
\hline 13 & M & 26 & 177.0 & 87.0 & Flex-Foot Flex-Walk & Sleeve & Trauma \\
\hline 14 & $\mathrm{~F}$ & 63 & 176.0 & 78.0 & Össur Ceterus & Pin & Cancer \\
\hline
\end{tabular}

$\mathrm{F}=$ female, $\mathrm{M}=$ male, pin suspension = suspension using gel liner with distal locking pin, $\mathrm{PVD}=$ peripheral vascular disease, sleeve suspension = suspension with gel liner and knee sleeve, supracondylar suspension = using removable medial brim.

Table 2.

Results for various degrees of flexibility F1-F5 (mean \pm standard deviation) for all subjects.

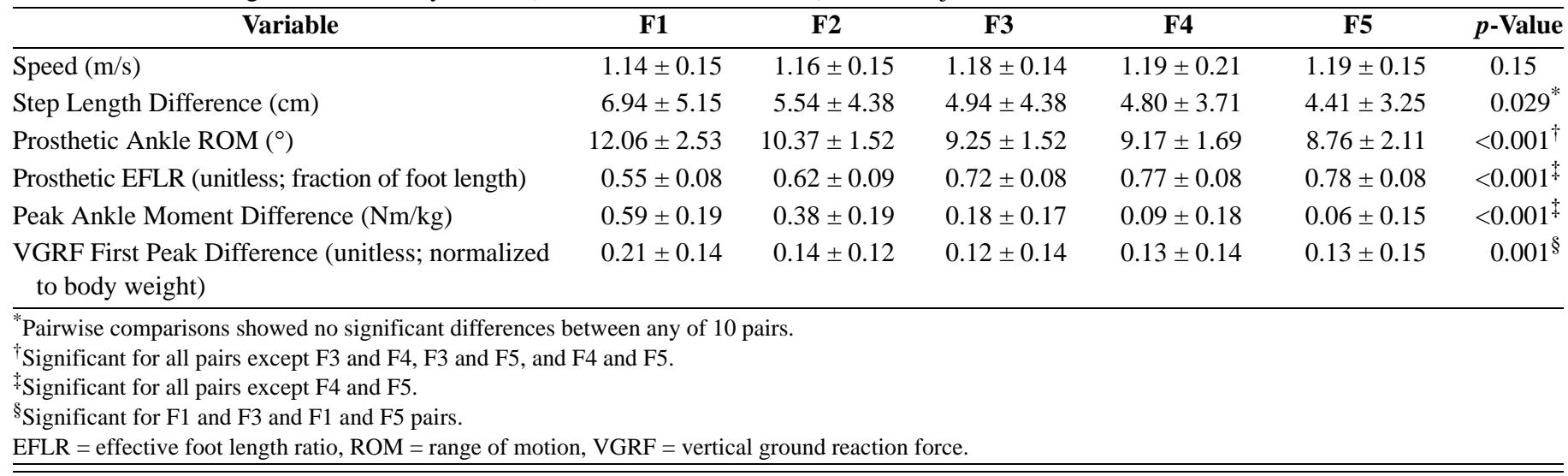


however, pairwise comparisons showed no significant differences between foot types.

The increase in prosthetic ankle ROM was as high as $3.3^{\circ}$ with increased forefoot flexibility $(p<0.001)$, supporting hypothesis 1 . Pairwise comparisons indicated significant differences in all pairs except F3 and F4, F3 and F5, and F4 and F5. The roll-over shapes for the five experimental prosthetic feet for a representative subject are shown in Figure 4. The roll-over shapes suggest a reduction in the forefoot moment arm with increasing flexibility. The increase in prosthetic EFLR was as high as 23 percent of the foot length with decreasing forefoot flexibility $(p<$ 0.001 ), supporting hypothesis 2 . Pairwise testing indicated differences in all pairs except the least flexible (F4 and F5). Figure 5 shows VGRFs and AMs for one representative subject. The differences in peak ankle flexion moments between the sound and prosthetic sides decreased as much as $0.53 \mathrm{Nm} / \mathrm{kg}$ as flexibility increased $(p<0.001)$, supporting hypothesis 3 . Post hoc testing indicated differences in all pairs except, again, the least flexible (F4 and F5). Difference in first peaks of the VGRF were as much as 9 percent of body weight when prosthetic feet with different forefoot flexibilities were used ( $p=0.001)$, supporting hypothesis 4 . Pairwise comparisons indicated significant differences between pairs F1 and F3 and F1 and F5.

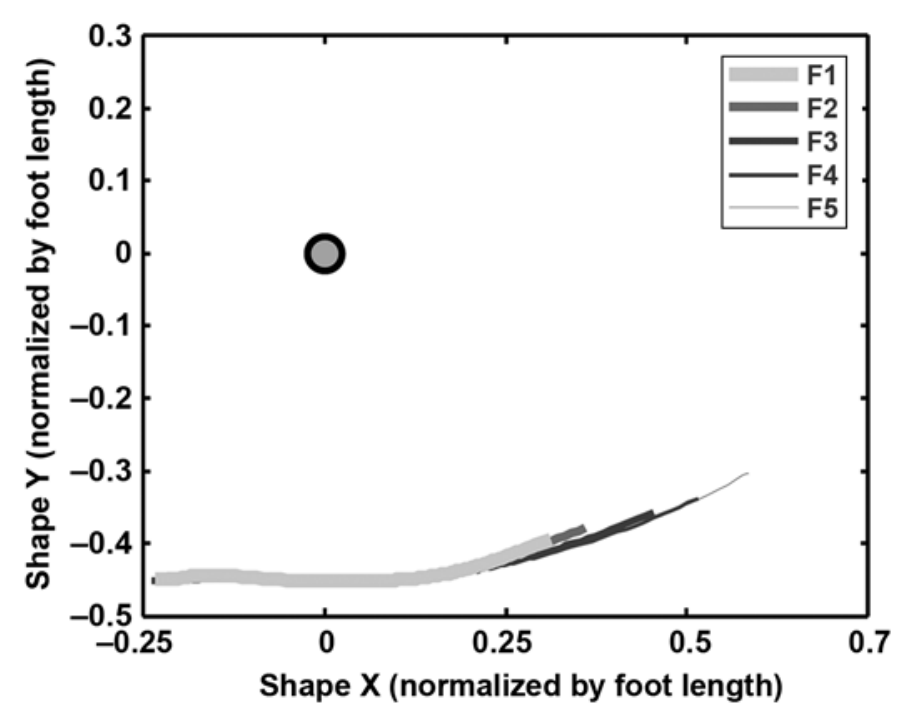

Figure 4.

Plot of roll-over shapes for five different feet (F1-F5) used by one representative subject (normalized by foot length). Each roll-over shape represents location of center of pressure of ground reaction force, transformed into coordinate system fixed to plate parallel to attachment surface of foot.

\section{DISCUSSION}

Results of this study support the idea that solid-ankle prosthetic feet with overly flexible forefoot sections do not allow the GRF to progress forward sufficiently, leading to reduced effective foot lengths, reduced prosthetic ankle moments, and a drop-off effect onto the sound limb. This drop-off leads to a noticeable limp, which is undesirable for cosmetic reasons, and may negatively affect sound limb health over time. The drop-off effect may also lead to increased oxygen consumption and/or increased demands of the muscles controlling the knee joint. Further work is needed to explore these ideas.

Although the results of this study support our hypotheses, they also suggest that a relatively wide range of forefoot flexibilities may be used without seeing major effects on gait. For example, no significant differences were seen in measured variables between the least flexible foot types (F4 and F5) and few differences were found between F3, F4, and F5. Large differences in forefoot stiffness properties may have existed between prosthetic feet F1, F2, and F3 and only small differences between prosthetic feet F3, F4, and F5. The EFLR results support this idea, with larger differences between F1, F2, and F3 compared with the differences between F3, F4, and F5. The range of EFLR values for the feet used in this study (0.55-0.78) is similar to that found earlier for a sample of commercially available prosthetic feet (0.63-0.81) (see Hansen et al. [9]).

In theory, the drop-off effect could lead to reduced step lengths on the sound side [8]. However, pairwise comparisons of step length difference did not indicate significant differences in this measure between any of the prosthetic foot types. Step length differences across subjects were highly variable. One potential explanation for inconsistent step length differences could be the use of compensatory mechanisms to adapt to the different flexibilities. Modeling work by Sessoms showed that step length is a function of many variables and that persons can easily adapt to keep step lengths equal, even with shortened effective foot lengths [16]. A trend toward reduced sound limb step lengths was found earlier for prosthetic feet with reduced arc lengths [8], but this trend also was not statistically significant.

The increase in prosthetic "ankle" ROM for feet with increased forefoot flexibility is highly logical and an expected result. However, the measurement was included in the study to allow comparison with results of earlier studies that found that high-profile carbon prosthetic feet allowing 

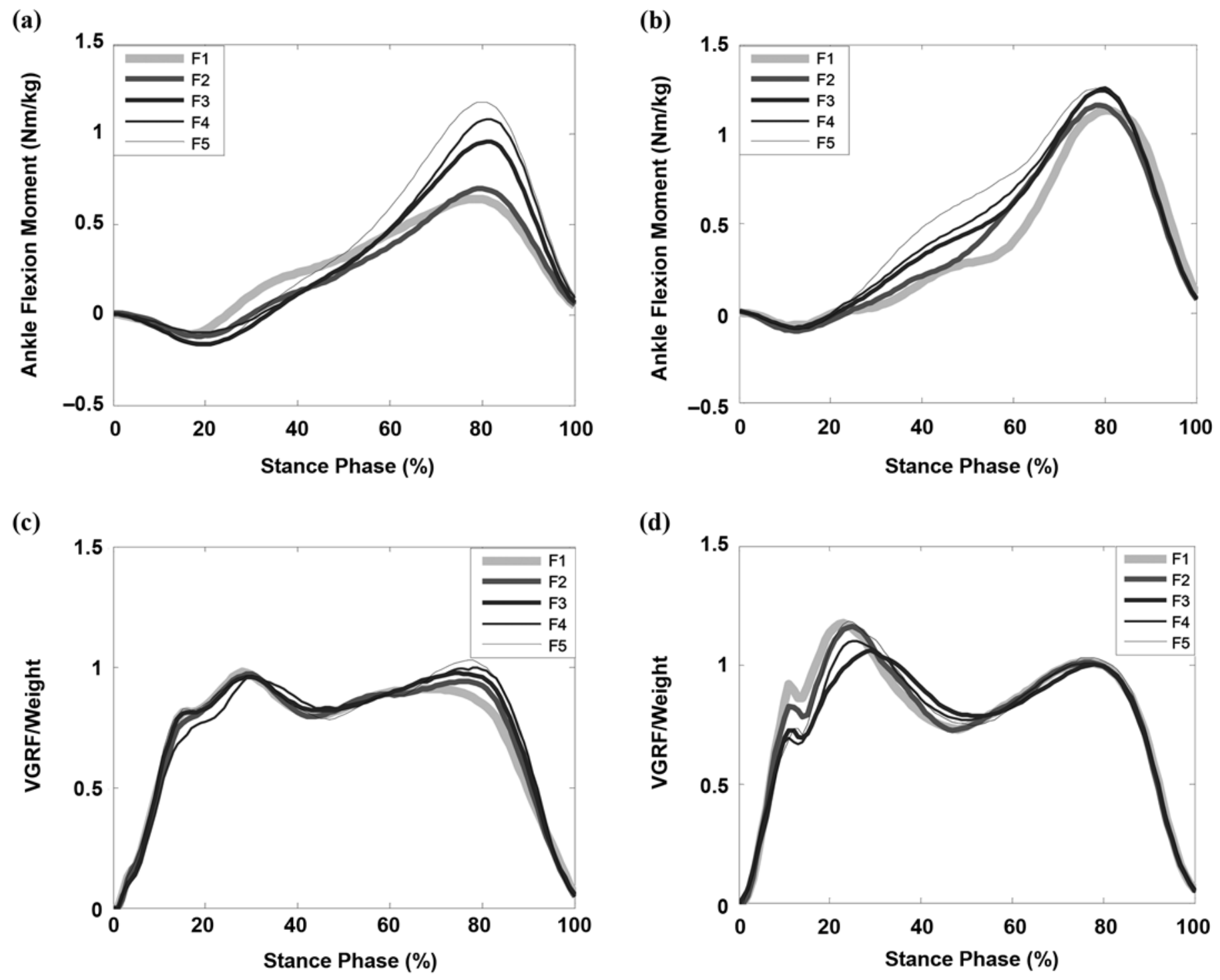

Figure 5.

External ankle moments of five different feet (F1-F5) for (a) prosthetic and (b) sound sides for one representative subject. Vertical ground reaction forces (VGRF) for (c) prosthetic and (d) sound sides. Forces are normalized to body weight. Each line represents different prosthetic foot for both prosthetic and sound sides. First peaks are evident at about 25\% stance phase.

increased ankle ROM also led to decreased sound limb loading in persons with unilateral transtibial amputation [5-6]. In contrast, the present study found increased sound limb loading when prosthetic ankle ROM increased when subjects used these solid-ankle feet. The high-profile carbon fiber foot used in previous studies included a prosthetic ankle-foot system with flexibility spanning from the socket to the end of the foot (a J-shaped carbon-fiber spring), while this study utilized experimental feet with rigid shanks and solid ankles, a factor potentially important to the different findings. We believe that the EFLR (the forefoot lever arm) of the different feet is critical in determining sound limb loading. When this anterior lever arm is shortened sufficiently, a dropoff occurs, leading to increased loads on the contralateral side [8-9].

Prosthetic feet with excessive rigidity (beyond those studied here) may possibly increase sound limb loading. The rocker-based inverted pendulum has been used by Gard and Childress to explain vertical excursions of the body during walking [17]. In reality, the rocker used by the body 
has a physical end that most likely affects the transition of load between feet. When rolling reaches the end of the rocker, the model can be thought to change to a simple inverted pendulum about the rocker end. The downward movement of a mass falling over a simple inverted pendulum is larger than the downward component of a mass on a rocker-based inverted pendulum (assuming similar lengths of the pendulum "legs"). This larger downward "falling" component of the mass could lead to the more forceful loading of the contralateral side as observed in this study. In prosthetic feet with excessive rigidity, the GRF would still progress very quickly to the end of the effective rocker, leading to a tipping movement and a potential drop-off effect. As an example, the data in Figure 5 suggest that prosthetic foot F3 provided the most balanced loading between limbs for this prosthesis user. The F3 prosthetic foot was designed with a forefoot flexibility that most closely mimicked the effective rocker radius created by the nondisabled ankle-foot system during walking.

Although the first peaks of the VGRF were most symmetric when subjects used the prosthetic foot designed to match the physiological ankle-foot roll-over shape (F3), subjects were still unbalanced. Data from Table 2 show that the sound limb still experienced a first-peak VGRF that was 12 percent of body weight higher on average than the prosthetic limb. Data from an earlier study of the effects of arc length on gait in which subjects used a similar series of experimental feet also yielded asymmetries in limb loading [8]. The difference may relate to several factors, including a lack of active push-off from the prosthetic feet in late stance phase and/or reduced energy storage and return from the prosthetic feet in this study. The work of Kuo et al. suggests that push-off from the trailing limb can reduce the work needed from the leading limb [18]. The passive nature of the prosthetic feet tested and the lack of push-off may have increased the work required of the leading limb, potentially accounting for the remaining asymmetry in first-peak VGRF. However, the prosthetic feet in this study more likely were not able to store and release the amount of energy required to yield symmetric VGRF between limbs. Prosthetic ankle-foot systems with flexible shanks, such as the Flex-Foot, may provide sufficient arc length, flexibility, and energy storage and return needed to provide a more symmetric loading pattern between limbs. In fact, Snyder et al. reported first peaks of the VGRFs that were actually increased on the prosthetic side compared with the sound side in persons with unilateral transtibial amputations using the Flex-Foot [6]. It is not clear if low- profile energy storage and return feet can yield similar results in loading symmetry.

One of the limitations of this study was the short accommodation time the subjects had with each prosthetic foot. The study was designed so that all research occurred inside the laboratory, but if subjects were permitted more accommodation time, they might have displayed different gait characteristics. A study using longer accommodation times could possibly show data relevant to long-term use of the different prosthetic foot designs.

In this study no exclusion criteria were used related to the subject's everyday prosthesis. Some subjects could have regularly used prosthetic feet that were stiffer than those used in the study, and therefore these subjects were not as experienced walking on prosthetic feet that had a softer forefoot. These subjects could have displayed a more asymmetric gait when walking on feet with greater flexibilities. Conversely, subjects that were habituated to walking on prosthetic feet with a more flexible forefoot may have exhibited a more asymmetric gait when walking on the feet that were stiffer.

Alignment was performed for each prosthetic foot used in the study. While keeping alignment constant would have provided a more standardized methodological approach, it would not have accurately reflected clinical practice. When the prosthetist aligned each subject's current prosthetic foot to their usual socket, he or she may have made adjustments that minimized the effect of differences between the feet. Earlier work suggests that prosthetists align prosthetic feet with different roll-over shapes toward a single, perhaps "ideal," roll-over shape for the individual [19]. Qualitative assessments of adjustments made in this study support this idea, and quantitative assessments are ongoing to determine if the roll-over shapes of the various feet were "nested" toward a single roll-over shape.

\section{CONCLUSIONS}

The solid-ankle prosthetic feet in this study with excessively flexible forefoot sections produced similar effects on gait of users with a unilateral transtibial prosthesis as prosthetic feet with short arc lengths. When prosthetic feet have excessively flexible forefoot sections, they provide shorter effective foot lengths, reducing ankle moments on the prosthetic side and leading to a "drop-off" effect when transitioning load from the prosthesis to the sound limb. 


\section{ACKNOWLEDGMENTS}

\section{Author Contributions:}

Study concept and design: A. Hansen.

Acquisition of data: E. Klodd, A. Hansen.

Prosthesis fitting: M. Edwards, S. Fatone.

Analysis and interpretation of data: E. Klodd, A. Hansen, S. Fatone, M. Edwards.

Drafting of manuscript: E. Klodd, A. Hansen.

Critical revision of manuscript for important intellectual content:

E. Klodd, A. Hansen, S. Fatone, M. Edwards.

Statistical analysis: E. Klodd, A. Hansen, S. Fatone.

Obtained funding: A. Hansen.

Study supervision: A. Hansen.

Financial Disclosures: The authors have declared that no competing interests exist.

Funding/Support: This material was based on work supported by Department of Veterans Affairs (VA) Rehabilitation Research and Development Service grant A4325R.

Additional Contributions: We acknowledge the assistance of Rebecca Stine, MS, in acquiring data for the study; Kerice Tucker, BS, in providing mechanical testing data for the feet; and Walter Afable, $\mathrm{CP}$, in referring research subjects. Data were collected within the VA Chicago Motion Analysis Research Laboratory of the Jesse Brown VA Medical Center, Chicago, Illinois.

Institutional Review: The research protocol was approved by both the Northwestern University Institutional Review Board and the Research and Development Committee of the Jesse Brown VA Medical Center. Participant Follow-Up: The authors do not plan to inform participants of the publication of this study.

\section{REFERENCES}

1. Czerniecki J. Research and clinical selection of foot-ankle systems. J Prosthet Orthot. 2005;17(4):S35-S37. DOI:10.1097/00008526-200510001-00012

2. Hofstad C, Linde H, Limbeek J, Postema K. Prescription of prosthetic ankle-foot mechanisms after lower limb amputation. Cochrane Database Syst Rev. 2004;(1):CD003978. [PMID: 14974050]

3. Van Der Linde H, Hofstad CJ, Geurts AC, Postema K, Geertzen JH, Van Limbeek J. A systematic literature review of the effect of different prosthetic components on human functioning with a lower-limb prosthesis. J Rehabil Res Dev. 2004;41(4):555-70. [PMID: 15558384] DOI:10.1682/JRRD.2003.06.0102

4. American Academy of Orthotists and Prosthetists. Official findings of the state-of-the-science conference on prosthetic foot/ankle mechanisms. J Prosthet Orthot. 2005;17(4S):1-52.

5. Powers CM, Torburn L, Perry J, Ayyappa E. Influence of prosthetic foot design on sound limb loading in adults with unilateral below-knee amputations. Arch Phys Med Rehabil. 1994;75(7):825-29. [PMID: 8024435]
6. Snyder RD, Powers CM, Fontaine C, Perry J. The effect of five prosthetic feet on the gait and loading of the sound limb in dysvascular below-knee amputees. J Rehabil Res Dev. 1995;32(4):309-15. [PMID: 8770795]

7. Wagner J, Sienko S, Supan T, Barth D. Motion analysis of SACH vs. Flex-Foot in moderately active below-knee amputees. Clin Prosthet Orthot. 1987;11(1):55-62.

8. Hansen AH, Meier MR, Sessoms PH, Childress DS. The effects of prosthetic foot roll-over shape arc length on the gait of trans-tibial prosthesis users. Prosthet Orthot Int. 2006;30(3):286-99. [PMID: 17162519] DOI:10.1080/03093640600816982

9. Hansen AH, Sam M, Childress DS. The effective foot length ratio (EFLR), a potential tool for characterization and evaluation of prosthetic feet. J Prosthet Orthot. 2004; 16(2):41-45. DOI:10.1097/00008526-200404000-00002

10. Sam M, Hansen AH, Childress DS. Characterisation of prosthetic feet used in low-income countries. Prosthet Orthot Int. 2004;28(2):132-40. [PMID: 15382807] DOI:10.1080/03093640408726698

11. Knox EH. The role of prosthetic feet in walking [dissertation]. [Evanston (IL)]: Northwestern University; 1996.

12. Hansen A, Childress D, Knox E. Rollover shapes of human locomotor systems: Effects of walking speed. Clin Biomech. 2004;19(4):407-14.

13. Kadaba MP, Ramakrishnan HK, Wootten ME. Measurement of lower extremity kinematics during level walking. J Orthop Res. 1990;8(3):383-92. [PMID: 2324857] DOI:10.1002/jor.1100080310

14. Winter DA. Biomechanics and motor control of human movement. New York (NY): Wiley; 1990.

15. Field AP. Discovering statistics using SPSS. 3rd ed. London (UK): Sage Publications; 2009.

16. Sessoms PH. Step by step: A study of step length in ablebodied persons, race walkers, and persons with amputations [dissertation]. [Evanston (IL)]: Northwestern University; 2008.

17. Gard SA, Childress DS. What determines the vertical displacement of the body during normal walking? J Prosthet Orthot. 2001;13(3):64-67. DOI:10.1097/00008526-200109000-00009

18. Kuo AD, Donelan M, Ruina A. Energetic consequences of walking like an inverted pendulum: Step-to-step transitions. Exerc Sport Sci Rev. 2005;33(2):88-97. [PMID: 15821430] DOI:10.1097/00003677-200504000-00006

19. Hansen AH, Meier MR, Sam M, Childress DS, Edwards ML. Alignment of trans-tibial prostheses based on roll-over shape principles. Prosthet Orthot Int. 2003;27(2):89-99.

[PMID: 14571938] DOI:10.1080/03093640308726664 
Submitted for publication October 15, 2009. Accepted in revised form June 9, 2010.

This article and any supplementary material should be cited as follows:

Klodd E, Hansen A, Fatone S, Edwards M. Effects of prosthetic foot forefoot flexibility on gait of unilateral transtibial prosthesis users. J Rehabil Res Dev. 2010; 47(9):899-910.

DOI:10.1682/JRRD.2009.10.0166

Crosser nemern

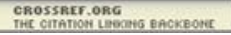


